\title{
Adiposity-Independent Effects of Aging on Insulin Sensitivity and Clearance in
}

\section{Humans and Mice}

Nicole Ehrhardt, ${ }^{1}$ Jinrui Cui, ${ }^{2}$ Sezin Dagdeviren, ${ }^{3}$ Suchaorn Saengnipanthkul, ${ }^{3}$ Helen S. Goodridge, ${ }^{4,5}$ Jason K. Kim, ${ }^{3,6}$ Louise Lantier, ${ }^{7}$ Xiuqing Guo, ${ }^{8}$ Yii-Der I. Chen, ${ }^{8}$ Leslie J. Raffel, ${ }^{9}$ Thomas A. Buchanan, ${ }^{10}$ Willa A. Hsueh, ${ }^{11}$ Jerome I. Rotter, ${ }^{8}$ Mark O. Goodarzi, ${ }^{2,12}$ and Miklós Péterfy ${ }^{1,5,12}$

${ }^{1}$ Department of Basic Medical Sciences, Western University of Health Sciences, Pomona, CA

${ }^{2}$ Division of Endocrinology, Diabetes, and Metabolism, Department of Medicine, CedarsSinai Medical Center, Los Angeles, CA

${ }^{3}$ Program in Molecular Medicine, University of Massachusetts Medical School, Worcester, MA

${ }^{4}$ Board of Governors Regenerative Medicine Institute, Cedars-Sinai Medical Center, Los Angeles, CA

${ }^{5}$ Department of Biomedical Sciences, Cedars-Sinai Medical Center, Los Angeles, CA

${ }^{6}$ Division of Endocrinology, Metabolism, and Diabetes, Department of Medicine, University of Massachusetts Medical School, Worcester, MA

${ }^{7}$ Department of Molecular Physiology and Biophysics, Vanderbilt University, Nashville, TN

${ }^{8}$ Institute for Translational Genomics and Population Sciences, Los Angeles BioMedical Research Institute and Department of Pediatrics, Harbor-UCLA Medical Center, Torrance, CA

${ }^{9}$ Department of Pediatrics, Division of Genetic and Genomic Medicine, University of California, Irvine, CA

${ }^{10}$ Department of Physiology and Biophysics and Department of Medicine, Keck School of Medicine of USC, Los Angeles, CA

${ }^{11}$ Division of Endocrinology, Diabetes and Metabolism, Department of Medicine, Wexner Medical Center, The Ohio State University, Columbus, $\mathrm{OH}$

${ }^{12}$ Department of Medicine, David Geffen School of Medicine at UCLA, Los Angeles, CA

M.O.G and M.P. contributed equally to this work.

Corresponding authors: Mark O. Goodarzi (Mark.Goodarzi@cshs.org) and Miklós Péterfy (mpeterfy@westernu.edu) Tel: +1 9097063949

Abstract word count: 263

Main text word count: 3,613

Tables: 3

Figures: 2 


\begin{abstract}
Aims/hypothesis: Aging is associated with impaired insulin sensitivity and increased prevalence of type 2 diabetes. However, it remains unclear whether aging-related insulin resistance is due to age per se, or increased adiposity associated with advanced age. In the present study, we investigate the impact of aging on insulin sensitivity independent of changes in body composition.

Methods: Cohorts of C57BL/6J male mice at 4-8 months of age ('young') and 18-27 mo ('aged') exhibiting similar body composition were characterized with static (plasma glucose and insulin levels) and dynamic (glucose and insulin tolerance tests) measures of glucose metabolism on chow and high-fat diets. Insulin sensitivity was assessed by hyperinsulinemic-euglycemic clamp analysis. The relationship between aging and insulin resistance in humans was investigated in 1,250 non-diabetic Mexican-American individuals who underwent hyperinsulinemic-euglycemic clamps.

Results: In mice with similar body composition, age had no detrimental effect on plasma glucose and insulin levels. However, aged mice demonstrated mildly, but reproducibly, improved glucose tolerance on both chow and high-fat diets due to increased glucosestimulated insulin secretion. Moreover, hyperinsulinemic-euglycemic clamps revealed impaired insulin sensitivity and reduced insulin clearance in aged mice on both diets. Consistent with results in the mouse, age remained an independent determinant of insulin resistance after adjustment for body composition in Mexican-Americn males. Advanced age was also associated with diminished insulin clearance, but this effect was dependent on increased BMI.
\end{abstract}


Conclusions/interpretation: This study demonstrates for the first time that aging per se impairs insulin sensitivity independent of adiposity in mice and humans. These results raise the possibility that the pathogenetic mechanisms of age-related and obesityassociated insulin resistance are distinct.

Keywords: aging, insulin sensitivity, insulin clearance

\section{Abbreviations}

BAI body adiposity index

GEE generalized estimating equations

HF high-fat

IQR interquartile range

MCRI metabolic clearance rate of insulin

T2D type 2 diabetes 


\section{INTRODUCTION}

The negative influence of aging on insulin sensitivity and the prevalence of type 2 diabetes (T2D) has long been recognized [1]. However, the pathogenetic mechanisms responsible for the age-related deterioration of glucose metabolism remain incompletely understood $[2,3]$. A principal difficulty is that aging is associated with numerous adverse physiological changes (e.g. altered body composition, reduced physical activity, sarcopenia, inflammation), which makes it difficult to decipher the role of aging itself from the impact of age-related comorbidities. In particular, as adiposity is a chief determinant of insulin sensitivity, the age-related accumulation of fat mass is a major confounder in studies investigating the metabolic effects of aging [4]. For example, whereas aging-associated declines in glucose tolerance and insulin sensitivity are well documented in the mouse, previous reports are based on comparisons between young and old mice with different body composition [5-8]. Thus, the impact of age per se on insulin sensitivity in the mouse is currently unknown.

A substantial body of evidence indicates that insulin sensitivity also declines with advanced age in humans [9-16]. To discriminate between the effects of age versus ageassociated changes in body composition, previous studies employed young and elderly cohorts matched for adiposity, or adjusted for this trait in population-based cohorts. Investigations in predominantly non-Hispanic white cohorts consistently failed to demonstrate an effect of aging on insulin sensitivity after adjustment for altered body composition suggesting that increased adiposity, and not age per se, is responsible for age-associated deterioration of glucose tolerance in this population [15-20]. In contrast, 
in a Japanese population-based cohort, age remained positively correlated with insulin resistance even after adjusting for fat content [21]. Remarkably, adjustment for adiposity revealed age-related improvement in insulin sensitivity in other cohorts with predominantly African-American participants [22-24]. Taken together, these observations suggest that genetic and/or other ethnicity-specific determinants may play a role in agerelated changes in insulin action and raise the possibility that impaired glucose homeostasis associated with aging may have different etiologies in different populations.

In the present study, we investigate whether aging-related insulin resistance is a direct effect of age, or a consequence of increased adiposity associated with aging in mouse and human subjects. To explore the adiposity-independent effects of aging on insulin action, we assess insulin sensitivity in young and aged mice with similar body composition for the first time. In human studies, we address this question in MexicanAmericans, a population in which the metabolic impact of aging has not been invetigated before despite their strong predisposition to diabetes [25]. 


\section{METHODS}

\section{Mice}

Young and aged male C57BL/6J mice were purchased from The Jackson Laboratory (Bar Harbor, ME) at 2-3 and 12 months of age, respectively, and maintained at Cedars-Sinai Medical Center until analysis at ages indicated in the figure legends. We used several approaches to ensure that only apparently healthy aged mice were included in our study. First, body weights were measured biweekly and mice showing weight loss at two successive time points were excluded from phenotyping. Second, complete blood cell count was evaluated with a HEMAVET 950 FS counter (Drew Scientific, FL) to identify and exclude mice with lymphomas, a prevalent neoplasm in aging C57BL/6 mice [26]. Finally, after phenotypic characterization, aged mice underwent necropsy and mice with visible tumors and enlarged organs were excluded from analysis. Animals were maintained under specific pathogen-free (SPF) conditions on a 14-h light/10-h dark cycle and fed ad libitum Laboratory Rodent Diet 5053 (LabDiet) or a high-fat (HF) diet (D12492; 60 kcal\% from lard; Research Diets) for 9 weeks with free access to water. Experimental procedures were approved by the Institutional Animal Care and Use Committees at Cedars-Sinai Medical Center, Western University, University of Massachusetts and Vanderbilt University.

\section{Mouse phenotyping}

Body composition was determined by quantitative magnetic resonance analysis (EchoMRI, TX). Blood glucose concentrations were measured with a OneTouch portable 
glucose meter (LifeScan, CA). Plasma insulin and C-peptide were assayed by ELISA (ALPCO, NH). In glucose tolerance tests (GTT), overnight fasted mice were retroorbitally injected with $1.5 \mathrm{~g} / \mathrm{kg}$ (chow diet) or $1 \mathrm{~g} / \mathrm{kg}$ (HF diet) glucose followed by blood glucose measurements. Insulin tolerance tests (ITT) were performed in ad libitum fed mice by retro-orbital injection of $0.8 \mathrm{U} / \mathrm{kg}$ (chow diet) or $1.2 \mathrm{U} / \mathrm{kg}$ (HF diet) insulin followed by blood glucose measurements. Hyperinsulinemic-euglycemic clamping of mice was performed at the National Mouse Metabolic Phenotyping Centers (MMPC) at the University of Massachusetts Medical School and Vanderbilt University (Supplemental Material). The metabolic clearance rate of insulin (MCRI) was calculated as insulin infusion rate divided by the mean plasma insulin concentration at steady state [27]. The calculation of MCRI assumes that endogenous insulin secretion is completely suppressed in the setting of hyperinsulinemic clamp, as has been demonstrated in several species [28-30].

\section{Human study subjects and phenotyping}

The current study was conducted in 1,250 non-diabetic subjects from two independent family-based cohorts recruited from the Los Angeles area, the Hypertension-Insulin Resistance (HTN-IR) and the Mexican-American Coronary Artery Disease (MACAD) cohorts. HTN-IR consists of Los Angeles Hispanic-American families ascertained via a proband with essential hypertension [31]. MACAD participants were drawn from adult offspring of probands with coronary artery disease and their spouses [32]. Participants were free of major medical illness and none were taking glucocorticoids or 
antihyperglycemic agents that could affect glucose homeostasis when they were phenotyped. All subjects gave written informed consent prior to participation.

\section{Human phenotyping}

We studied 579 subjects from HTN-IR and 671 MACAD participants who had undergone phenotyping with the hyperinsulinemic-euglycemic clamp as described previously [33]. The glucose infusion rate (M value) during the last 30 minutes of steadystate glucose and insulin levels during the clamp reflects glucose uptake by all tissues of the body (mainly insulin-mediated glucose uptake in muscle) and is directly correlated with tissue insulin sensitivity [34]. The insulin sensitivity index (M/I) was calculated as M divided by the steady state plasma insulin level (I). The MCRI was calculated as the insulin infusion rate divided by the insulin concentration during the steady state of the euglycemic clamp, as previously described [34, 35].

Body adiposity index (BAI) was calculated as (hip circumference in centimeters $) /(\text { height in meters })^{1.5}-18$ ) [36]. BMI was calculated as weight in kilograms divided by height in meters squared.

All studies were approved by Human Subjects Protection Institutional Review Boards at UCLA, the University of Southern California and Cedars-Sinai Medical Center.

\section{Statistical analysis}


In the mouse data, normally distributed values are expressed as mean \pm SEM and nonnormally distributed values are represented by the median and interquartile range (IQR). Comparisons between two groups were performed by Student's t-test or Mann-Whitney U test. GTT and ITT results were analyzed by 2-way repeated measures ANOVA and Holm-Sidak post hoc tests. ANOVA and correlation analyses of non-normally distributed variables were performed after transformation or on ranks. Outlier data points were defined as those falling outside $2 x I Q R$. Based on this criterion, 2 extreme values were excluded from analysis in the entire study (1 young and 1 aged mouse in Figure $2 \mathrm{G}$ ). Differences at $\mathrm{p}<0.05$ were considered statistically significant.

In the human data, log-transformed (BMI, fasting glucose, fasting insulin) or square root-transformed ( $\mathrm{M}$ value, $\mathrm{M} / \mathrm{I}, \mathrm{MCRI})$ trait values were used to normalize the distribution for statistical analyses. Generalized estimating equations (GEE) were used to assess the relationships between pairs of traits (univariate analyses) or joint effects of multiple traits (multivariate analyses) on $\mathrm{M}, \mathrm{M} / \mathrm{I}$, or $\mathrm{MCRI}$, adjusting for familial relationships. The weighted GEE1 [37] was computed assuming an exchangeable correlation structure and using the sandwich estimator of the variance to account for familial correlation present in family data. GEE was used to derive standardized regression coefficients, which in any one regression equation are measured on the same scale, with a mean of zero and a standard deviation of one. They are then directly comparable to one another, with the largest coefficient indicating which independent variable has the greatest association with the dependent variable. 


\section{RESULTS}

\section{Aging is Associated with Improved Glucose Tolerance, and Reduced Insulin Sensitivity and Clearance in Mice}

Several previous studies investigating the metabolic impact of aging documented reduced glucose tolerance and insulin resistance in old mice (14-26 months of age) relative to young (1-3 months) mice [5-8]. However, as body fat content increases during the first few months of life, these studies were confounded with different body compositions in old vs young mice and the metabolic effects of age per se remained unclear [5-8]. As body weight and adiposity reach a plateau in mature adult ( $\sim 4$ months) mice $[38,39]$, we reasoned that using mouse cohorts beyond this age would allow metabolic characterization without the confounding effects of altered body composition. Indeed, mature adult C57BL/6J mice at 4-5 months of age (from here on referred to as 'young') exhibited body weight, lean mass, fat mass and percent fat content that were indistinguishable from 18-21-month-old ('aged') mice (Fig. 1A). Furthermore, young and aged mice maintained similar fasting and ad libitum fed glucose and insulin levels (Fig. $1 \mathrm{~B}$ and $1 \mathrm{C})$. Intravenous glucose tolerance tests (GTT) revealed that aged mice were slightly, but significantly more glucose tolerant than young mice (Fig. 1D). This was due, at least in part, to increased glucose-stimulated insulin secretion in aged mice, as both insulin and C-peptide levels were elevated in this group 15 min after glucose injection (Fig. 1E and 1F), consistent with previous studies [40]. In insulin tolerance tests (ITT), young and aged mice demonstrated similar glucose response to intravenously-injected 
insulin (Fig. 1G). To confirm these results, we characterized a second group of young (6month-old) and aged (25-month-old) mice with similar body composition (Supplemental Fig. 1A). Consistent with results obtained in the initial cohort, aged mice exhibited improved glucose tolerance (Supplemental Fig. 1B), but unchanged insulin tolerance (Supplemental Fig. 1C) relative to young mice.

We further investigated the metabolic impact of aging by assessing glucose homeostasis in a third cohort of young (8-month-old) and aged (27-month-old) male mice by hyperinsulinemic-euglycemic clamp analyses. Body weight and composition, fasting plasma glucose and insulin levels, and the rate of hepatic glucose production were indistinguishable between young and aged mice in the basal state (Supplemental Table 1). However, despite identical rates of insulin infusion, aged mice exhibited elevated plasma insulin levels (Fig. 1H) and a lower rate of insulin clearance from the circulation (Fig. 1I) during the clamp. No significant differences were observed in glucose infusion rate, whole body glucose turnover, suppression of hepatic glucose production, and glucose uptake into muscle and adipose tissue under clamp conditions (Fig. 1J-M, Supplemental Table 1, Supplemental Fig. 2A-B). Based on the observation of similar insulin action despite higher insulin levels in aged animals, we conclude that aging is associated with increased hepatic, muscle and adipose insulin resistance in male mice independent of changes in adiposity.

Aging is Associated with Improved Glucose Tolerance, but Impaired Muscle and Adipose Insulin Sensitivity and Reduced Insulin Clearance in Diet-Induced Obese

\section{Mice}


The results in lean mice prompted us to investigate whether diet-induced metabolic stress may exacerbate aging-related insulin resistance. To test this hypothesis, we assessed the impact of aging on glucose homeostasis in the context of diet-induced obesity by characterizing young (6-month-old) and aged (24-month-old) male C57BL/6J mice fed a HF diet (60 kcal\% from fat) for 9 weeks. At the time of metabolic analysis young and aged mice had similar body weights and composition (Fig. 2A). Unexpectedly, aged mice maintained lower fasting and fed glucose levels (Fig. 2B) despite similar plasma insulin concentrations (Fig. 2C). Consistent with results obtained in lean mice, obese aged mice also demonstrated improved glucose tolerance relative to young animals (Fig. 2D). Furthermore, assessment of insulin tolerance by ITT revealed that exogenous insulin had a greater glucose-suppressing effect in aged mice at later time-points of the assay, although the difference in areas under the curve did not reach statistical significance (Fig. 2E). To evaluate insulin sensitivity directly, we performed hyperinsulinemic-euglycemic clamp experiments. Consistent with results on chow diet, obese aged mice maintained significantly higher insulin levels (Fig. 2F) and demonstrated reduced insulin clearance (Fig. 2G) during the clamp. Despite elevated plasma insulin concentration in aged mice, glucose infusion rate, whole body glucose turnover and glucose uptake in muscle and adipose were similar in young and aged mice (Fig. 2H-K, Supplemental Table 2, Supplemental Fig. 2C-D). However, insulin-mediated suppression of hepatic glucose production was significantly greater in aged mice (Fig. 2I). Taken together, our results indicate that in the metabolic context of diet-induced obesity, aging is associated with improved glucose tolerance, reduced insulin clearance, and impaired muscle and adipose insulin sensitivity in male mice. 


\section{Sex-specific Effect of Aging on Insulin Sensitivity and Clearance in Mexican-}

\section{Americans}

To explore the relationship between age, insulin resistance, insulin clearance and adiposity in humans, we performed correlation analyses in a Mexican-American cohort of 1,250 participants free of diabetes and overt metabolic disease. The age range of the cohort is 7 decades (16-87 years) and insulin sensitivity has been assessed by hyperinsulinemic-euglycemic clamp. Univariate correlation analyses revealed agingassociated changes in glucose homeostasis including elevated fasting plasma glucose levels, reduced insulin sensitivity ( $\mathrm{M}$ and $\mathrm{M} / \mathrm{I})$ and decreased MCRI (Table 1). To assess body composition, we used BMI and BAI, an alternative surrogate measure of fat content more closely tracking percent fat content in Mexican-Americans [36]. Both BMI and BAI exhibited strong positive correlations with age indicating fat accumulation during aging in this population (Table 1).

Sex-specific analysis demonstrated significant negative correlations between age and insulin action in both males and females with notably weaker effects in the latter (Table 2). To separate the contributions of age and adiposity on insulin action, we performed multivariate correlation analyses with measures of insulin sensitivity as dependent variables, and age and body composition as independent variables. In males, age remained strongly correlated with insulin resistance even after adjustment for changes in body composition (Table 2). In contrast, controlling for adiposity reduced or abolished the correlation between age and insulin action in females, depending on the measure of insulin sensitivity used (Table 2). While the effect of age was relatively 
modest in the multivariate models tested (Table 2), BMI and BAI had robust effect sizes and remained strongly negatively correlated with insulin sensitivity after controlling for age in both sexes (Supplemental Table 3). Taken together, these results demonstrate an age-dependent decline of insulin sensitivity in Mexican-American males and females. In females, this is principally due to aging-associated increases in fat mass, whereas age is an independent determinant of insulin resistance in males.

Sex-specific analysis of insulin clearance revealed that the age-related decline observed in the full cohort (Table 1) was exclusively due to males, as significant correlation between age and MCRI could not be detected in females (Table 3). Furthermore, multivariate analysis indicated that the aging-associated decrease of insulin clearance in males was due to increased body fat content and not age per se (Table 3). 


\section{DISCUSSION}

The association of aging with impaired insulin resistance has been well established in mice [5-8]. However, as body composition changes with normal aging, previous studies have been unable to disentangle the effects of aging per se from those of increased adiposity. In the present study, we address this problem for the first time by characterizing mice of different age, but similar body composition. We took advantage of the fact that body weight and fat content in C57BL/6 mice reach a plateau at the mature adult stage ( $\sim 4$ months) and remain unchanged thereafter $[38,39]$. Thus, using 'young' mouse cohorts at $>4$ months of age allowed us to investigate the adiposity-independent effects of aging on glucose metabolism for the firat time.

When mice with similar body compositions were compared, advanced age had no detrimental impact on static (i.e. plasma glucose and insulin levels) or dynamic measures (i.e. GTT, ITT) of glucose metabolism. In fact, we found that aged mice exhibited slightly improved glucose tolerance, an observation that was replicated in three independent mouse cohorts on two different diets. Consistent with these results, improved glucose tolerance in aging $\mathrm{C} 57 \mathrm{BL} / 6 \mathrm{~J}$ mice has also been previously reported [40]. Importantly and similar to the present study, Leiter et al. used 'young' mice at the relatively advanced age of 4.5- to 5 month, which likely diminished differences in body composition between young and old mice, although adiposity was not directly assessed in that study [40]. It has also been demonstrated that aging is associated with increased pancreatic islet area and insulin content, but unaffected $\beta$-cell sensitivity to glucose [40]. 
These results are consistent with our observation of elevated C-peptide levels after glucose administration in aged mice and suggest that elevated glucose-stimulated insulin secretion contributes to their improved glucose tolerance. Another likely contributing factor is reduced insulin clearance in old mice. We hypothesize that the interaction between increased production and reduced clearance of insulin may explain the apparent over-compensation for insulin resistance in aged mice. In conclusion, while glucose intolerance is a hallmark of aging [7, 41], our results demonstrate that this is not an inevitable consequence of advanced age, but most likely due to increased adiposity associated with aging in $\mathrm{C} 57 \mathrm{BL} / 6 \mathrm{~J}$ mice.

We assessed the impact of aging on insulin sensitivity by hyperinsulinemiceuglycemic clamps in young and aged mice with similar body compositions. Despite similar rates of insulin infusion, aged mice exhibited significantly higher plasma insulin concentration during the clamp. This observation held true for both lean mice maintained on a normal chow diet and diet-induced obese mice on a HF-diet. In lean mice, measures of whole-body and tissue insulin action were similar in young and aged animals. Together with elevated insulin levels in the latter, these data demonstrate for the first time that aging per se impairs insulin sensitivity in $\mathrm{C} 57 \mathrm{BL} / 6 \mathrm{~J}$ mice.

We initially hypothesized that the metabolic stress associated with HF diet feeding would exacerbate aging-related impairments in glucose homeostasis. However, our results do not support this hypothesis. In fact, obese aged mice maintained lower basal glucose levels and exhibited improved glucose tolerance relative to their young counterparts. Furthermore, we unexpectedly found that aging impacted tissues differently in diet-induced obese mice. Consistent with observations in lean mice, despite elevated 
clamp insulin levels, insulin action was similar in muscle and adipose tissues of young and aged animals indicating relative insulin resistance in the latter. In contrast, suppression of hepatic glucose production was substantially stronger in obese aged versus young mice. Although the concurrent elevation of insulin levels complicates the interpretation of this observation, our results suggest that hepatic insulin sensitivity may not be adversely impacted by age in the context of diet-induced obesity. Consistent with this conclusion, aging has also been associated with improved hepatic insulin action in humans in a handful of studies where hepatic glucose production was determined, [22, 42].

Most previous human studies focusing predominantly on non-Hispanic white populations led to the consensus view that aging-associated insulin resistance is due to age-related increase in adiposity rather than age per se [15-20, 22]. In the current study, we addressed this issue in a cohort of non-Hispanic white subjects for the first time. Consistent with previous reports, insulin sensitivity declined with age in both sexes. However, the relative contributions of adiposity and age to aging-associated insulin resistance were different in the sexes. In Mexican-American females, diminished insulin action was due to increases in adiposity and not age itself, in line with results in Caucasians. In contrast, while elevated adiposity was a major determinant of declining insulin action in males, age also contributed to insulin resistance independently of changes in body composition. A similar conclusion was reached in a recent study in Japanese subjects [21]. Taken together, these results highlight the previously underappreciated role of sex and ethnicity in aging-associated insulin resistance in humans. 
Previous studies investigating the impact of aging on insulin clearance yielded mixed results $[9,15,43-45]$. Here we report consistent age-related declines in the rate of insulin removal from the circulation in mouse and human subjects. Nonetheless, the underlying mechanisms appear to be different in the two species. Whereas increased adiposity is the main driver of reduced insulin turnover in aging Mexican-Americans, diminished insulin clearance in aged $\mathrm{C} 57 \mathrm{BL} / 6 \mathrm{~J}$ mice is independent of body composition. This observation is consistent with the recent demonstration of age-related loss of hepatic endothelial fenestrations and diminished insulin uptake associated with reduced insulin clearance in mice and rats [46]. Our results also raise the question of whether age-related changes in insulin clearance are the consequence of aging per se or secondary to insulin resistance. As aging is associated with impaired insulin sensitivity in both MexicanAmericans and the mouse model we used, further studies will be required to discriminate between these possibilities.

In summary, the present study provides evidence in mouse and human populations that aging impairs insulin sensitivity independently of altered body composition. Based on the analysis of a Mexican-American cohort, this conclusion contrasts with previous results obtained in non-Hispanic white populations and suggests a role for genetic factors in age-related metabolic dysfunction. The present work also highlights the impact of sex on insulin resistance, as age was an independent contributor to this trait only in Mexican-American men, but not women. As our mouse study was limited to the characterization of $\mathrm{C} 57 \mathrm{BL} / 6 \mathrm{~J}$ males, future studies involving females and additional mouse strains will be needed to investigate the role of sex and genetic determinants in age-related changes in insulin sensitivity. An intriguing possibility raised 
by the present study is that the mechanisms of obesity- and aging-associated insulin resistance may be distinct, a notion consistent with a previous report [47]. This possibility may also have important therapeutic implications for the treatment of T2D in the aging population. As demonstrated in the present study, male C57BL/6J mice represent a suitable animal model to investigate the molecular mechanisms underlying the adiposityindependent effects of aging on insulin resistance. 
Acknowledgements. The authors thank Drs. Maura Rossetti, Xiao Z. Shen, Tuantuan Zhao, Shuang Chen and Sujin Suk for their contributions to this project.

Funding. This study was supported by National Institutes of Health grants R01HL088457， R01-DK079888，R01-HL67974，P30-DK063491，P50-HL55005， M01RR000425, M01-RR000043, UL1-TR000124, U2C-DK093000, U2C-DK059637 and P30- DK020593.

Duality of Interest. The authors declare no conflict of interest.

Author Contributions. N.E. conducted experiments, analyzed data and revised the manuscript. J.C. analyzed data. S.D. and S.S. conducted experiments. H.S.G. provided materials and advised experiments. J.K.K. and L.L. supervised experiments, analyzed data and revised the manuscript. X.G., Y.I.C., L.J.R., T.A.B., W.A.H. and J.I.R acquired data. M.O.G. and M.P. conceived of the study design, supervised the study, analyzed data, and revised the manuscript. M.P. wrote the manuscript. All authors approved the final version of the manuscript. M.O.G. and M.P. are guarantors of this work and, as such, had full access to all the data in the study and take responsibility for the integrity of the data and the accuracy of the data analysis. 


\section{REFERENCES}

[1] Halter JB, Musi N, McFarland Horne F, et al. (2014) Diabetes and cardiovascular disease in older adults: current status and future directions. Diabetes 63: 2578-2589

[2] Davidson MB (1979) The effect of aging on carbohydrate metabolism: a review of the English literature and a practical approach to the diagnosis of diabetes mellitus in the elderly. Metabolism 28: 688-705

[3] Chang AM, Halter JB (2003) Aging and insulin secretion. Am J Physiol Endocrinol Metab 284: E7-12

[4] Barzilai N, Huffman DM, Muzumdar RH, Bartke A (2012) The critical role of metabolic pathways in aging. Diabetes 61: 1315-1322

[5] Bailey CJ, Flatt PR (1982) Hormonal control of glucose homeostasis during development and ageing in mice. Metabolism 31: 238-246

[6] Gonzalez-Rodriguez A, Mas-Gutierrez JA, Mirasierra M, et al. (2012) Essential role of protein tyrosine phosphatase $1 \mathrm{~B}$ in obesity-induced inflammation and peripheral insulin resistance during aging. Aging Cell 11: 284-296

[7] Houtkooper RH, Argmann C, Houten SM, et al. (2011) The metabolic footprint of aging in mice. Sci Rep 1: 134

[8] Ropelle ER, Pauli JR, Cintra DE, et al. (2013) Targeted disruption of inducible nitric oxide synthase protects against aging, S-nitrosation, and insulin resistance in muscle of male mice. Diabetes 62: 466-470 
[9] Chen M, Bergman RN, Pacini G, Porte D, Jr. (1985) Pathogenesis of age-related glucose intolerance in man: insulin resistance and decreased beta-cell function. $\mathrm{J}$ Clin Endocrinol Metab 60: 13-20

[10] Defronzo RA (1979) Glucose intolerance and aging: evidence for tissue insensitivity to insulin. Diabetes 28: 1095-1101

[11] Chen M, Bergman RN, Porte D, Jr. (1988) Insulin resistance and beta-cell dysfunction in aging: the importance of dietary carbohydrate. J Clin Endocrinol Metab 67: $951-957$

[12] Houmard JA, Weidner MD, Dolan PL, et al. (1995) Skeletal muscle GLUT4 protein concentration and aging in humans. Diabetes 44: 555-560

[13] Petersen KF, Befroy D, Dufour S, et al. (2003) Mitochondrial dysfunction in the elderly: possible role in insulin resistance. Science 300: 1140-1142

[14] Short KR, Vittone JL, Bigelow ML, et al. (2003) Impact of aerobic exercise training on age-related changes in insulin sensitivity and muscle oxidative capacity. Diabetes 52: 1888-1896

[15] Basu R, Breda E, Oberg AL, et al. (2003) Mechanisms of the age-associated deterioration in glucose tolerance: contribution of alterations in insulin secretion, action, and clearance. Diabetes 52: 1738-1748

[16] Basu R, Dalla Man C, Campioni M, et al. (2006) Effects of age and sex on postprandial glucose metabolism: differences in glucose turnover, insulin secretion, insulin action, and hepatic insulin extraction. Diabetes 55: 2001-2014 
[17] Coon PJ, Rogus EM, Drinkwater D, Muller DC, Goldberg AP (1992) Role of body fat distribution in the decline in insulin sensitivity and glucose tolerance with age. $\mathrm{J}$ Clin Endocrinol Metab 75: 1125-1132

[18] Ferrannini E, Vichi S, Beck-Nielsen H, Laakso M, Paolisso G, Smith U (1996) Insulin action and age. European Group for the Study of Insulin Resistance (EGIR). Diabetes 45: 947-953

[19] Karakelides H, Irving BA, Short KR, O'Brien P, Nair KS (2010) Age, obesity, and sex effects on insulin sensitivity and skeletal muscle mitochondrial function. Diabetes 59: 89-97

[20] Utzschneider KM, Carr DB, Hull RL, et al. (2004) Impact of intra-abdominal fat and age on insulin sensitivity and beta-cell function. Diabetes 53: 2867-2872

[21] Oya J, Nakagami T, Yamamoto Y, et al. (2014) Effects of age on insulin resistance and secretion in subjects without diabetes. Intern Med 53: 941-947

[22] Lalia AZ, Dasari S, Johnson ML, et al. (2016) Predictors of Whole-Body Insulin Sensitivity Across Ages and Adiposity in Adult Humans. J Clin Endocrinol Metab 101: 626-634

[23] Chandler-Laney PC, Phadke RP, Granger WM, et al. (2011) Age-related changes in insulin sensitivity and beta-cell function among European-American and AfricanAmerican women. Obesity (Silver Spring) 19: 528-535

[24] Hunter GR, Chandler-Laney PC, Brock DW, Lara-Castro C, Fernandez JR, Gower BA (2010) Fat distribution, aerobic fitness, blood lipids, and insulin sensitivity in African-American and European-American women. Obesity (Silver Spring) 18: 274-281 
[25] Spanakis EK, Golden SH (2013) Race/ethnic difference in diabetes and diabetic complications. Curr Diab Rep 13: 814-823

[26] Blackwell BN, Bucci TJ, Hart RW, Turturro A (1995) Longevity, body weight, and neoplasia in ad libitum-fed and diet-restricted C57BL6 mice fed NIH-31 open formula diet. Toxicol Pathol 23: 570-582

[27] Ader M, Stefanovski D, Kim SP, et al. (2014) Hepatic insulin clearance is the primary determinant of insulin sensitivity in the normal dog. Obesity (Silver Spring) 22: $1238-1245$

[28] Edgerton DS, Kraft G, Smith M, et al. (2017) Insulin's direct hepatic effect explains the inhibition of glucose production caused by insulin secretion. JCI Insight 2: e91863

[29] Farmer TD, Jenkins EC, O'Brien TP, et al. (2015) Comparison of the physiological relevance of systemic vs. portal insulin delivery to evaluate whole body glucose flux during an insulin clamp. Am J Physiol Endocrinol Metab 308: E206-222

[30] Liljenquist JE, Horwitz DL, Jennings AS, Chiasson JL, Keller U, Rubenstein AH (1978) Inhibition of insulin secretion by exogenous insulin in normal man as demonstrated by C-peptide assay. Diabetes 27: 563-570

[31] Xiang AH, Azen SP, Raffel LJ, et al. (2001) Evidence for joint genetic control of insulin sensitivity and systolic blood pressure in hispanic families with a hypertensive proband. Circulation 103: 78-83

[32] Goodarzi MO, Guo X, Taylor KD, et al. (2003) Determination and use of haplotypes: ethnic comparison and association of the lipoprotein lipase gene and coronary artery disease in Mexican-Americans. Genet Med 5: 322-327 
[33] Labadzhyan A, Cui J, Peterfy M, et al. (2016) Insulin Clearance Is Associated with Hepatic Lipase Activity and Lipid and Adiposity Traits in Mexican Americans. PLoS One 11: e0166263

[34] DeFronzo RA, Tobin JD, Andres R (1979) Glucose clamp technique: a method for quantifying insulin secretion and resistance. Am J Physiol 237: E214-223

[35] Goodarzi MO, Taylor KD, Guo X, et al. (2005) Variation in the gene for musclespecific AMP deaminase is associated with insulin clearance, a highly heritable trait. Diabetes 54: 1222-1227

[36] Bergman RN, Stefanovski D, Buchanan TA, et al. (2011) A better index of body adiposity. Obesity (Silver Spring) 19: 1083-1089

[37] Zeger SL, Liang KY (1986) Longitudinal data analysis for discrete and continuous outcomes. Biometrics 42: 121-130

[38] Lessard-Beaudoin M, Laroche M, Demers MJ, Grenier G, Graham RK (2015) Characterization of age-associated changes in peripheral organ and brain region weights in C57BL/6 mice. Exp Gerontol 63: 27-34

[39] Fischer KE, Hoffman JM, Sloane LB, et al. (2016) A cross-sectional study of male and female C57BL/6Nia mice suggests lifespan and healthspan are not necessarily correlated. Aging (Albany NY) 8: 2370-2391

[40] Leiter EH, Premdas F, Harrison DE, Lipson LG (1988) Aging and glucose homeostasis in C57BL/6J male mice. FASEB J 2: 2807-2811

[41] Ayala JE, Samuel VT, Morton GJ, et al. (2010) Standard operating procedures for describing and performing metabolic tests of glucose homeostasis in mice. Dis Model Mech 3: 525-534 
[42] Broughton DL, James OW, Alberti KG, Taylor R (1991) Peripheral and hepatic insulin sensitivity in healthy elderly human subjects. Eur J Clin Invest 21: 13-21

[43] Ahren B, Pacini G (1998) Age-related reduction in glucose elimination is accompanied by reduced glucose effectiveness and increased hepatic insulin extraction in man. J Clin Endocrinol Metab 83: 3350-3356

[44] Minaker KL, Rowe JW, Tonino R, Pallotta JA (1982) Influence of age on clearance of insulin in man. Diabetes 31: 851-855

[45] Lorenzo C, Hanley AJ, Wagenknecht LE, et al. (2013) Relationship of insulin sensitivity, insulin secretion, and adiposity with insulin clearance in a multiethnic population: the insulin Resistance Atherosclerosis study. Diabetes Care 36: 101-103

[46] Mohamad M, Mitchell SJ, Wu LE, et al. (2016) Ultrastructure of the liver microcirculation influences hepatic and systemic insulin activity and provides a mechanism for age-related insulin resistance. Aging Cell 15: 706-715

[47] Bapat SP, Myoung Suh J, Fang S, et al. (2015) Depletion of fat-resident Treg cells prevents age-associated insulin resistance. Nature 528: 137-141 
Table 1. Clinical characteristics and their correlation with age in Mexican-Americans

\begin{tabular}{|c|c|c|c|c|c|}
\hline & $\mathrm{n}$ & Median & IQR & r & $\mathrm{p}$ \\
\hline Age [years] & 1250 & 34 & 15 & & \\
\hline BMI $\left[\mathrm{kg} / \mathrm{m}^{2}\right]$ & 1250 & 28.2 & 6.5 & 0.188 & $<0.0001$ \\
\hline BAI [\%] & 1250 & 31.8 & 8.7 & 0.199 & $<0.0001$ \\
\hline Fasting plasma glucose $[\mathrm{mg} / \mathrm{dl}]$ & 1246 & 92.0 & 12.0 & 0.213 & $<0.0001$ \\
\hline Fasting plasma insulin $[\mu \mathrm{U} / \mathrm{ml}]$ & 1194 & 12.0 & 8.2 & 0.017 & 0.568 \\
\hline $\mathrm{M}\left[\mathrm{mg} / \mathrm{min} / \mathrm{m}^{2}\right]$ & 1250 & 223 & 150 & -0.205 & $<0.0001$ \\
\hline $\mathrm{M} / \mathrm{I}\left[\mathrm{mg} \times \mathrm{ml} / \mathrm{min} / \mathrm{m}^{2} / \mathrm{mU}\right]$ & 1191 & 1.72 & 1.43 & -0.192 & $<0.0001$ \\
\hline $\operatorname{MCRI}\left[\mathrm{ml} / \mathrm{m}^{2} / \mathrm{min}\right]$ & 1191 & 459 & 135 & -0.062 & 0.020 \\
\hline
\end{tabular}

The cohort consists of $42.8 \%$ males and $57.2 \%$ females. IQR, interquartile range; BMI, body mass index; BAI, body adiposity index; $M$, glucose disposal rate in the final 30 min of euglycemic clamp normalized by body surface area; M/I, M value divided by steady-state insulin concentration during the clamp; MCRI, metabolic clearance rate of insulin. $p<0.05$ values appear in bold. 


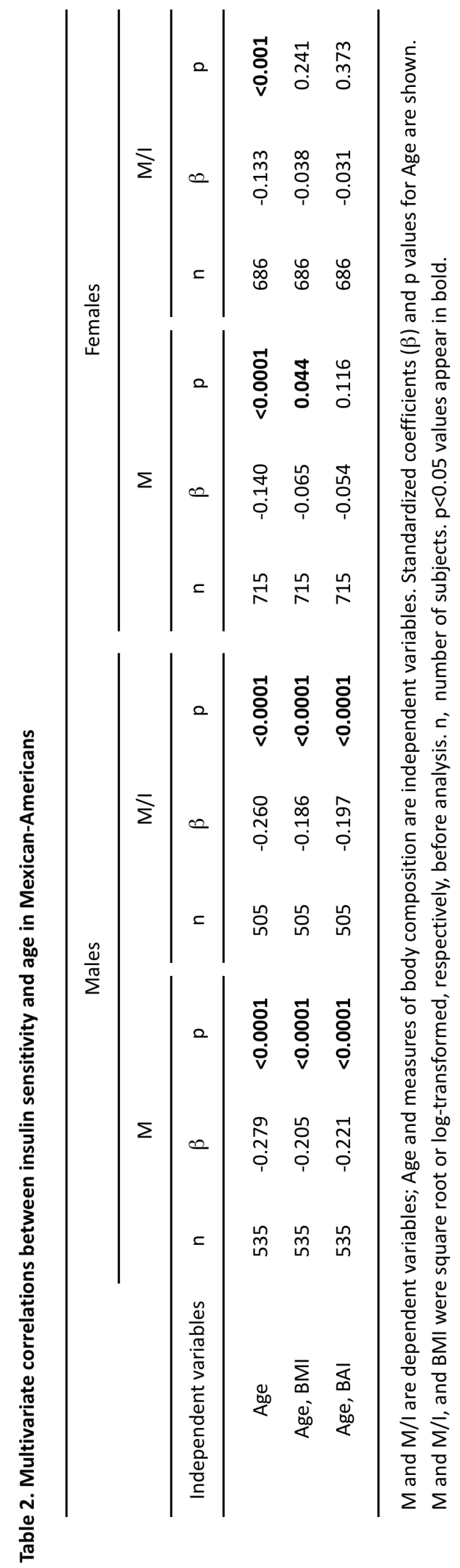


bioRxiv preprint doi: https://doi org/10.1101/333997- this version posted May 30 2018. The copyright holder for this preprint (which was not certified by peer review) is the author/funder, who has granted bioRxiv a license to display the preprint in perpetuity. It is made available under aCC-BY-NC-ND 4.0 International license.

Table 3. Multivariate correlations between insulin clearance and age in Mexican-Americans

\begin{tabular}{|c|c|c|c|c|c|c|}
\hline \multirow[b]{2}{*}{ Independent variables } & \multicolumn{3}{|c|}{ Males } & \multicolumn{3}{|c|}{ Females } \\
\hline & $\mathrm{n}$ & $\beta$ & $p$ & $\mathrm{n}$ & $\beta$ & $p$ \\
\hline Age & 505 & -0.104 & 0.008 & 686 & -0.033 & 0.335 \\
\hline Age, BMI & 505 & -0.068 & 0.112 & 686 & 0.029 & 0.422 \\
\hline Age, BAl & 505 & -0.069 & 0.107 & 686 & 0.023 & 0.527 \\
\hline
\end{tabular}

MCRI is the dependent variable; Age and measures of body composition are independent variables. Standardized coefficients $(\beta)$ and $p$ values for Age are shown. MCRI and BMI were square root or log-transformed, respectively, before analysis. $n$, number of subjects. $p<0.05$ values appear in bold. 


\section{FIGURE LEGENDS}

Figure 1 - Aging is associated with improved glucose tolerance, decreased insulin clearance and impaired insulin sensitivity in male $\mathrm{C} 57 \mathrm{BL} / 6 \mathrm{~J}$ mice on a chow diet. $A$ : Body weight (BW) and composition of young (4-5 months) and aged (18-21 months) mice ( $\mathrm{n}=9$ mice per group). Vertical axis shows mass in grams for BW, Lean and Fat, or body composition as percentage of fat for Fat\%. B: Blood glucose levels in overnight fasted and ad libitum fed mice ( $\mathrm{n}=9$ mice per group). $C$ : Plasma insulin levels in overnight fasted and ad libitum fed mice (horizontal bars represent median, $\mathrm{n}=7-10$ mice per group). $D$ : Glucose tolerance test ( $\mathrm{n}=9$ mice per group). ${ }^{*} \mathrm{P}<0.05$, comparison by $2-$ way repeated measures ANOVA (left panel) or Student's $t$ test (right panel). E: Plasma insulin levels 15 minutes after glucose injection (horizontal bars represent mean, $\mathrm{n}=6-7$ mice per group). ${ }^{*} \mathrm{P}<0.05$, comparison by Student's $t$-test. $F$ : Plasma C-peptide levels 15 minutes after glucose injection (horizontal bars indicate mean, $\mathrm{n}=6-7$ mice per group). ${ }^{*} \mathrm{P}<0.05$, comparison by Student's $t$-test. $G$ : Insulin tolerance test $(\mathrm{n}=9$ mice per group). $H$ : Plasma insulin levels during hyperinsulinemic-euglycemic clamp in young ( 8 months) and aged (27 months) mice (horizontal bars represent median, $\mathrm{n}=11-12$ mice per group). ${ }^{*} \mathrm{P}<0.05$, comparison by Mann-Whitney $\mathrm{U}$ test. $I$ : Metabolic clearance rate of insulin (MCRI) during the clamp (horizontal bars represent median, $\mathrm{n}=11-12$ mice per group). ${ }^{*} \mathrm{P}<0.05$, comparison by Mann-Whitney $\mathrm{U}$ test. $J$ : Glucose infusion rate (GIR) during the clamp ( $\mathrm{n}=11-12$ mice per group). $K$ : Suppression of hepatic glucose production during the clamp $(\mathrm{n}=11-12$ mice per group). Glucose uptake in skeletal 
muscle $(L)$ and adipose tissue $(M)$ during the clamp $(\mathrm{n}=11-12$ mice per group). Data are presented as mean $\pm \mathrm{SEM}$ unless stated otherwise.

Figure 2 - Aging is associated with improved glucose tolerance, decreased insulin clearance and impaired insulin sensitivity in male diet-induced obese C57BL/6J mice. $A$ : Body weight (BW) and composition of young (6 months) and aged (24 months) mice (n $=8$ mice per group) after 9 weeks of HF diet feeding. Vertical axis shows mass in grams for BW, Lean and Fat, or body composition as percentage of fat for Fat \%. B: Blood glucose levels in 5-hour fasted and ad libitum fed mice $(\mathrm{n}=6-8$ mice per group). $* \mathrm{P}<$ 0.05 , comparison by Student's $t$-test. $C$ : Plasma insulin levels in overnight fasted and $a d$ libitum fed mice (horizontal bars represent median, $\mathrm{n}=5-8$ mice per group). $D$ : Glucose tolerance test ( $\mathrm{n}=8$ mice per group). ${ }^{*} \mathrm{P}<0.05$, comparison by 2 -way repeated measures ANOVA (left panel) or Student's $t$ test (right panel). $E$ : Insulin tolerance test (n $=8$ mice per group). ${ }^{*} \mathrm{P}<0.05$, comparison by 2 -way repeated measures ANOVA. F: Plasma insulin levels during hyperinsulinemic-euglycemic clamp (horizontal bars represent median, $\mathrm{n}=5-7$ mice per group). ${ }^{*} \mathrm{P}<0.05$, comparison by Mann-Whitney $\mathrm{U}$ test. $G$ : Metabolic clearance rate of insulin (MCRI) during the clamp (horizontal bars represent median, $\mathrm{n}=5-7$ mice per group). ${ }^{*} \mathrm{P}<0.05$, comparison by Mann-Whitney $\mathrm{U}$ test. $H$ : Glucose infusion rate (GIR) during the clamp ( $\mathrm{n}=6-8$ mice per group). I: Suppression of hepatic glucose production during the clamp ( $\mathrm{n}=5-7$ mice per group). $* \mathrm{P}<0.05$, comparison by Student's $t$-test. Glucose uptake in skeletal muscle $(J)$ and adipose tissue $(K)$ during the clamp $(n=6-8$ mice per group). Data are presented as mean \pm SEM unless stated otherwise. 


\section{Figure 1}

A

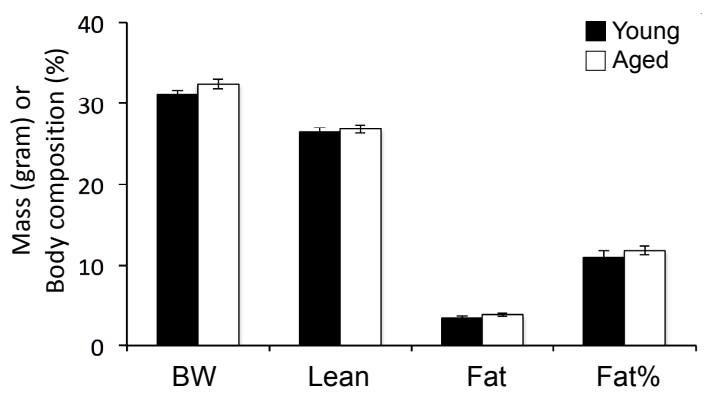

D

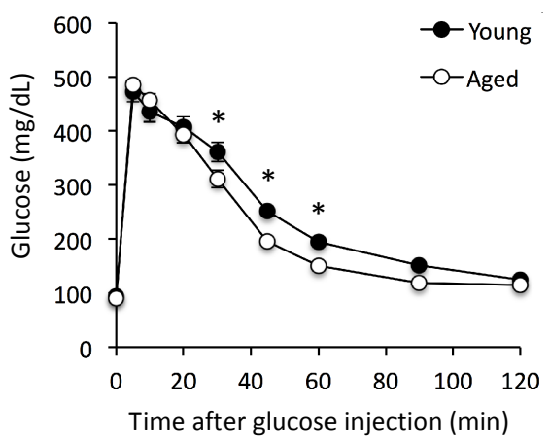

G

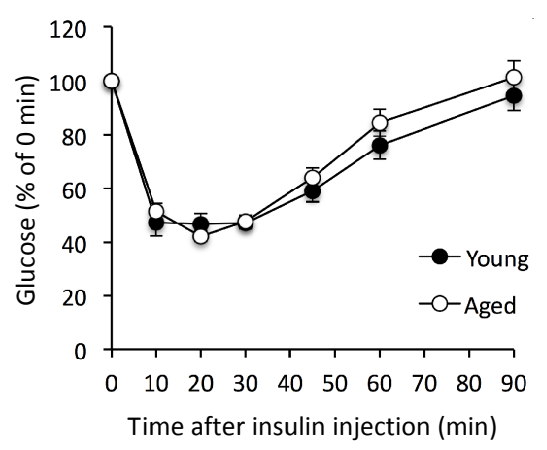

B

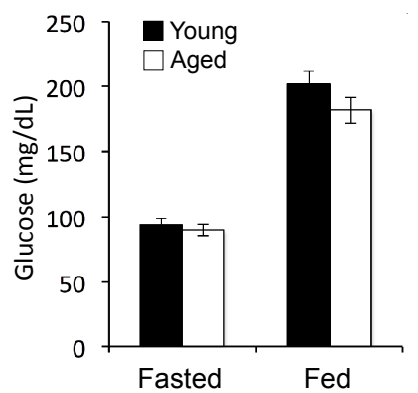

E
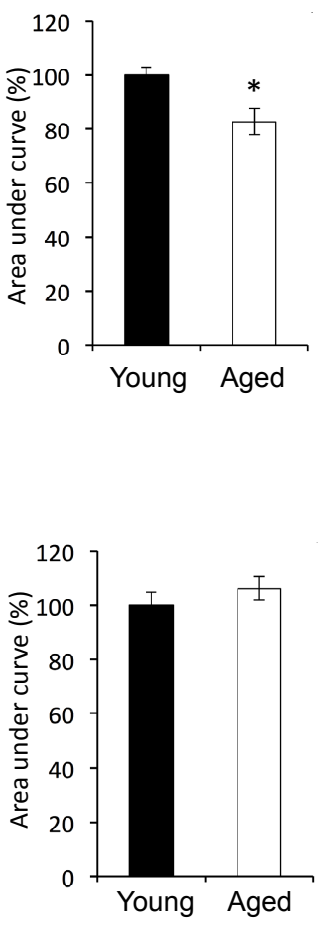

$\mathrm{H}$

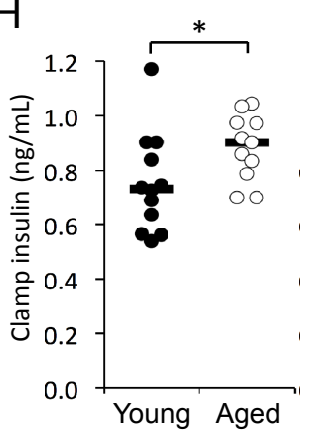

C

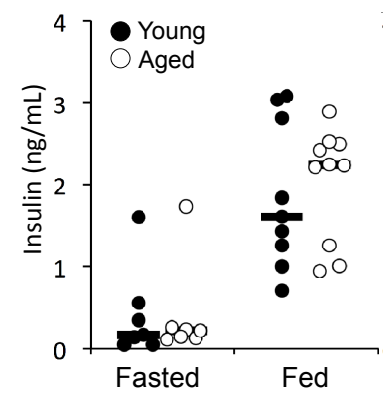

F
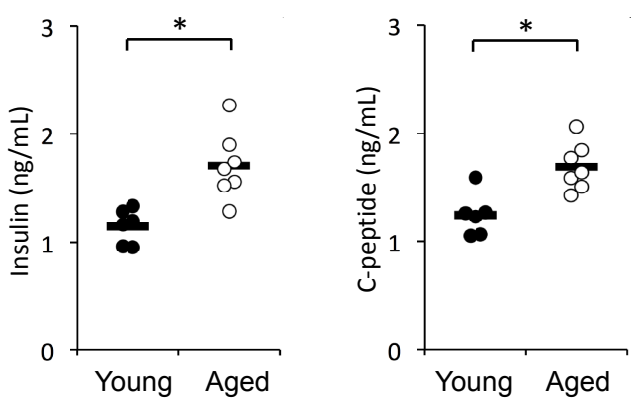

I

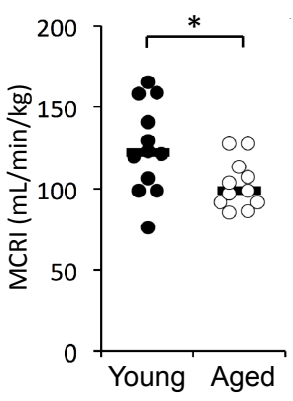

J

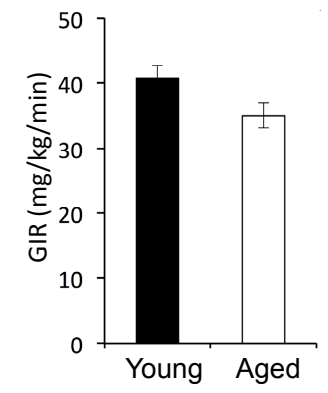

K

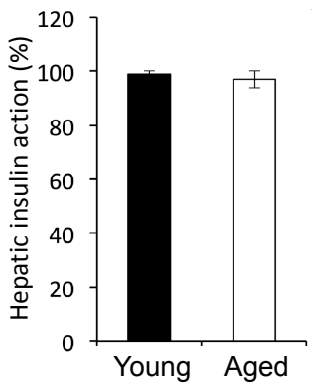

L

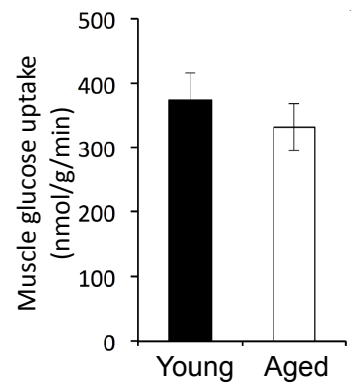

M

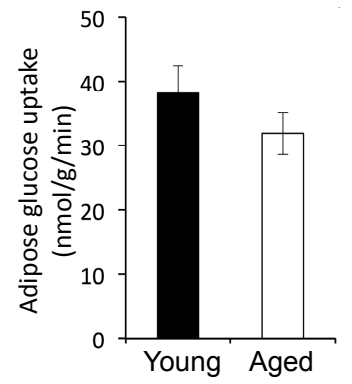


Figure 2

A

B

C
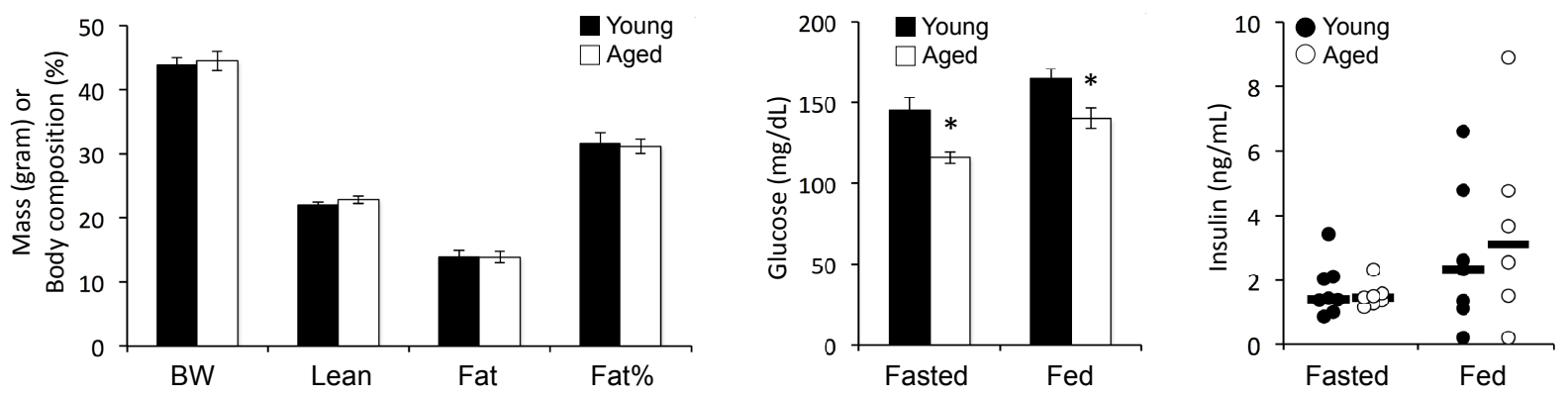

D
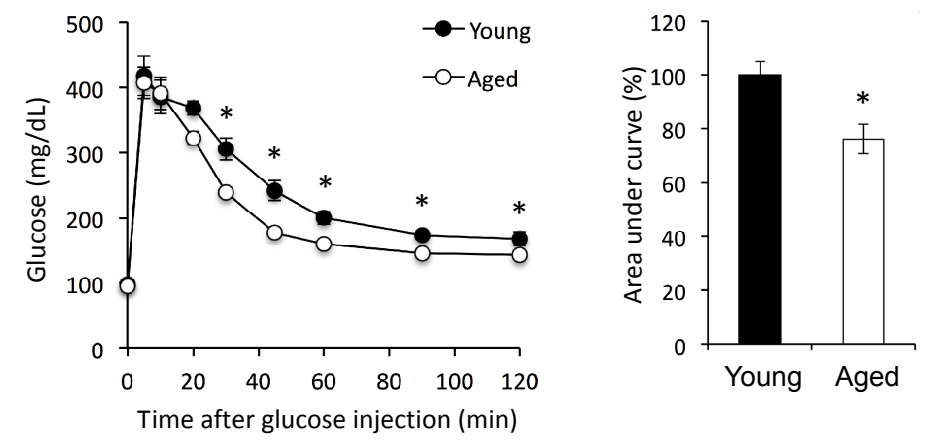

$E$

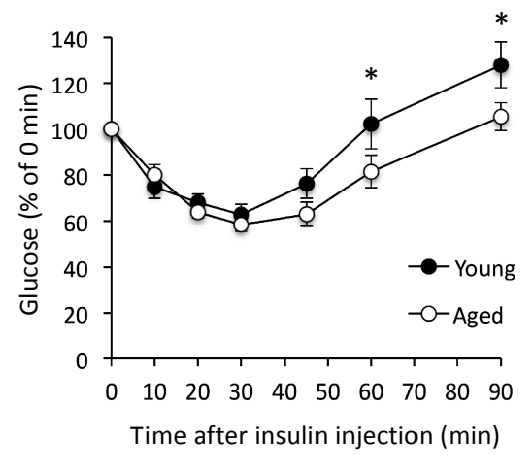

F

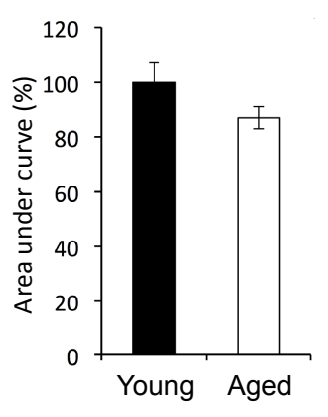

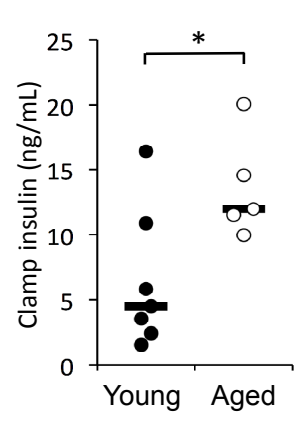

$G$

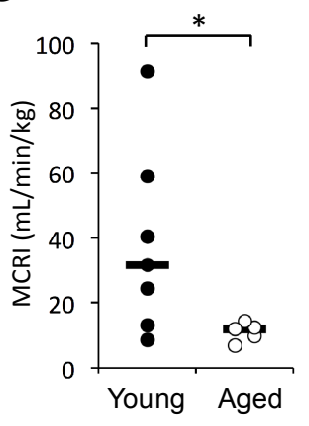

$\mathrm{H}$

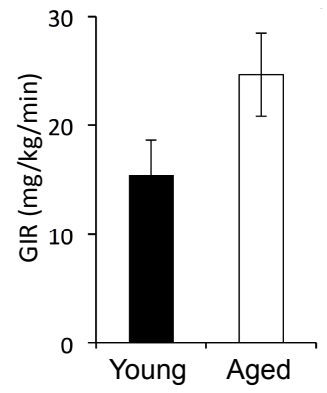

I

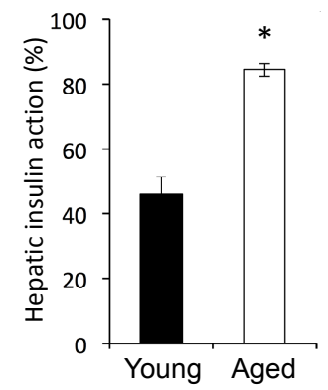

J

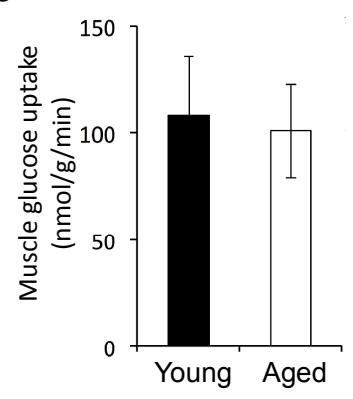

K

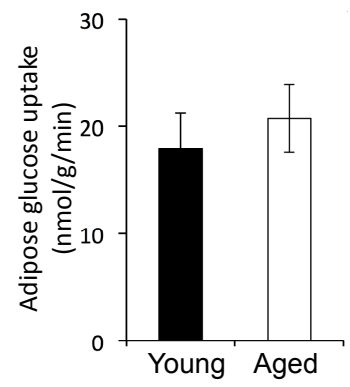

